Open Access

\title{
Application of transcranial direct current stimulation to psychiatric disorders: trends and perspectives
}

Yuma Yokoi ${ }^{*}$ and Tomiki Sumiyoshi

\author{
* Correspondence: \\ yyokoi@ncnp.go.jp \\ 'Department of Psychiatry, National \\ Center Hospital, National Center of \\ Neurology and Psychiatry, 4-1-1 \\ Ogawa-Higashi, Kodaira, Tokyo \\ 187-8553, Japan \\ Full list of author information is \\ available at the end of the article
}

\begin{abstract}
Background: Growing attention is paid to transcranial direct current stimulation (tDCS) as a novel neuromodulation method in the treatment of psychiatric illnesses. In spite of its simple procedure, electrophysiological influence of tDCS is complex and not fully understood. Therefore, its procedure and clinical application is yet to be established. To address this issue, we reviewed and summarized reports currently available, and proposed future directions.
\end{abstract}

Methods: We searched PubMed for the literature of tDCS, targeting depression, cognitive enhancement or schizophrenia, sham-controlled, and repeated stimulation sessions.

Results: Among psychiatric conditions, depression is most associated with positive effects of tDCS, based on the recent systematic review, due to homogeneity in methodology adopted in randomized sham-controlled trials. For cognitive enhancement and/or treatment of schizophrenia, results are less consistent, and the methods are more heterogeneous.

Conclusion: Large-scale well-designed trials are needed to more accurately evaluate the efficacy of tDCS. In this article, considerations of optimal stimulation conditions are also provided.

Keywords: Transcranial direct current stimulation, Neuromodulation, Depression, Dementia, Cognitive function, Schizophrenia

\section{Introduction}

Growing attention is paid to transcranial direct current stimulation (tDCS) as a novel neuromodulation method in the treatment of psychiatric illnesses. Its basic concept is simple; applying a weak current (no more than $2 \mathrm{~mA}$ ) over a period of time (usually no more than $30 \mathrm{~min}$ ) to the brain via two or more electrodes, of which at least one is put over the scalp. The history of tDCS can be traced back to the 19th century. In 1930s, tDCS was once disregarded as a treatment tool with the advent of electroconvulsive therapy. The reasons for its abandonment may also have included inconsistent results, unknown mode of action, and the lack of reliable and reproducible methods and verification processes (Steinberg 2013). However, the recent progress in methodology, reproducibility, and positive results under some conditions has facilitated the renaissance of tDCS. Among the neuromodulation devices currently available, e.g.

(C) 2015 Yokoi and Sumiyoshi. This is an Open Access article distributed under the terms of the Creative Commons Attribution License (http://creativecommons.org/licenses/by/4.0), which permits unrestricted use, distribution, and reproduction in any medium provided the original work is properly credited. The Creative Commons Public Domain Dedication waiver (http:// creativecommons.org/publicdomain/zero/1.0/) applies to the data made available in this article, unless otherwise stated. 
electroconvulsive therapy (ECT), vagus nerve stimulation, and transcranial magnetic stimulation (TMS) (Table 1), we will provide a brief overview of tDCS. Considerations will be given to the difference from other modalities of stimulation methods, as well as challenges to promote its clinical applications.

The most common type of $\mathrm{tDCS}$ is the $1 \mathrm{x} 1$ paradigm, which uses two (one anode and one cathode) rubber electrodes covered by saline-soaked sponges (usually 20 to $35 \mathrm{~cm}^{2}$ ).

Figure 1 shows an example of how applying tDCS to a subject looks like. In terms of safety, recent tDCS studies largely adapt the protocol including the use of sponges of 25 $35 \mathrm{~cm}^{2}$ in size and currents of $1-2 \mathrm{~mA}$ applied for up to $30 \mathrm{~min}$. Similar to the case with ECT, the mechanism of action of tDCS is not fully understood yet. There has been a concept that the electrical current through the brain is believed to evoke depolarization of neurons and increasing excitability of the cortical region below the anodal electrode, with opposite effects on the cathodal electrode (Stagg et al. 2013). However, controversy exists on this view (Lopez-Alonso et al. 2014; Wiethoff et al. 2014). Its excitatory effect remains up to $90 \mathrm{~min}$ (Nitsche and Paulus 2001), and daily repetitive stimulations elicit prolonged influences (Alonzo et al. 2012). Besides the effect on resting membrane potentials, tDCS has been suggested to modify synaptic transmissions linked to glutamatergic and GABAergic activities (Mondino et al. 2014; Stagg and Nitsche 2011). Specifically, the after-effects of tDCS have been demonstrated to depend on modifications of NMDAreceptor efficacy (Stagg and Nitsche 2011). The tDCS-induced excitability change is modulated by agonists or antagonists at catecholamine, acetylcholine or serotonin receptors (Stagg and Nitsche 2011; Medeiros et al. 2012).

Contrary to its simple procedure, current flows during tDCS seem complex. Compared to TMS, electric fields caused by tDCS tend to be more diffused and non-focal (Russell et al. 2013), and interpersonal anatomical difference may affect its clinical outcome along with change in its electrical field (Kim et al. 2014). In a computer model experiment, the electric field is influenced by geometry of sulci and gyri between electrodes (Miranda et al. 2013). Another report suggests thicknesses of the cerebrospinal

Table 1 Psychiatric neuromodulation methods compared with tDCS (adapted from (George et al. 2011))

\begin{tabular}{|c|c|c|c|c|c|}
\hline & ECT & VNS & TMS & DBS & tDCS \\
\hline \multirow{3}{*}{$\begin{array}{l}\text { Clinical Approval } \\
\text { By FDA }\end{array}$} & Depression & Epilepsy & TRD & PD & \multirow[t]{3}{*}{ None approved } \\
\hline & $\begin{array}{l}\text { Schizophrenia } \\
\text { etc. }\end{array}$ & TRD & Migraine & TRD & \\
\hline & & & & OCD & \\
\hline Anesthesia & General & General & No & General & No \\
\hline \multirow[t]{3}{*}{ Possible Risks } & Amnesia & Hoarseness & Headache & Stroke & Skin redness \\
\hline & Delirium & Cough & Dizziness & Infection & \multirow[t]{2}{*}{ Scalp discomfort } \\
\hline & & & $\begin{array}{l}\text { Scalp } \\
\text { discomfort }\end{array}$ & Seizure & \\
\hline \multirow[t]{2}{*}{$\begin{array}{l}\text { Number of sessions } \\
\text { for depression }\end{array}$} & $\begin{array}{l}8 \text { to } 12 \\
\text { sessions }\end{array}$ & NA & $\begin{array}{l}20 \text { to } 30 \\
\text { sessions }\end{array}$ & NA & 5 to 15 sessions \\
\hline & $\begin{array}{l}2 \text { to } 3 \text { sessions } \\
\text { a week }\end{array}$ & & $\begin{array}{l}3-5 \text { sessions } \\
\text { a week }\end{array}$ & & $\begin{array}{l}3-5 \text { sessions a } \\
\text { week }\end{array}$ \\
\hline $\begin{array}{l}\text { General cost in } \\
\text { the U.S. }\end{array}$ & $\begin{array}{l}\$ 800 \text { to } \$ 1000 \\
\text { per session }\end{array}$ & $\begin{array}{l}\$ 5,000 \\
\text { to } \$ 30,000\end{array}$ & $\$ 300$ per session & $\begin{array}{l}\$ 130,000 \\
\text { (Stroupe et al. 2014) }\end{array}$ & $\begin{array}{l}\text { No more than } \\
\text { TMS per session }\end{array}$ \\
\hline
\end{tabular}

ECT: electroconvulsive therapy, VNS: vagus nerve stimulation, TMS: transcranial magnetic stimulation, tDCS: transcranial direct current stimulation, TRD: treatment-resistant depression, PD: Parkinson's disease, OCD: Obsessive-compulsive disorder, NA: not applicable 


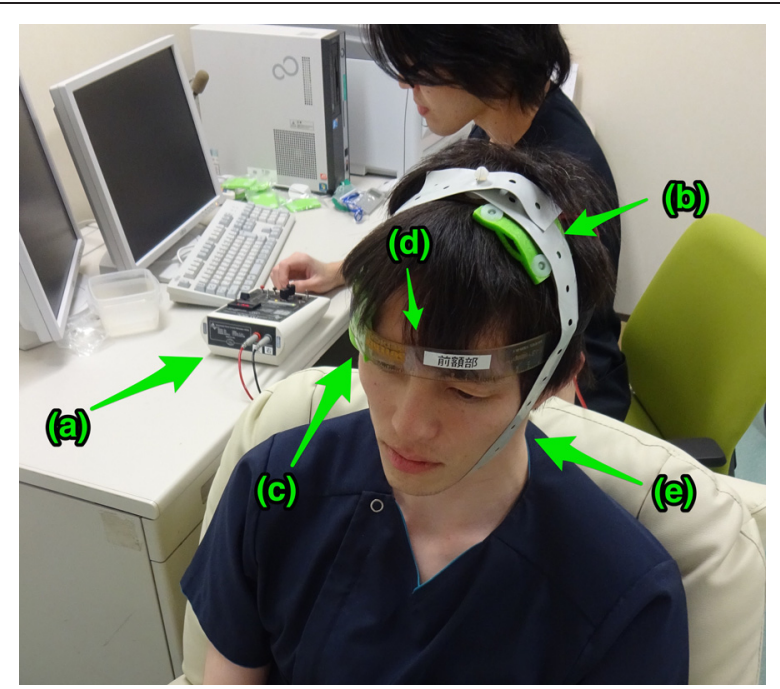

Fig. 1 tDCS administration at National Center of Neurology and Psychiatry Hospital. A subject (front) sits on a sofa relaxed, and a researcher (behind) controls the tDCS device (a). In this picture, anodal (b) and cathodal (c) electrodes with $35-\mathrm{cm}^{2}$ size are put on $\mathrm{F} 3$ and right supraorbital region, respectively. We use a head strap (d) for convenience and reproducibility, and also use a rubber band (e) for reducing resistance

fluid and the skull, the gyral depth, and the distance to the anode and cathode affect electric field, accounting for up to $50 \%$ of the spatial variation (Opitz et al. 2015). Moreover, even a slight (about $5 \%$ ) drift of electrodes during stimulation can cause significant change in electric field.(Woods et al. 2014) Therefore, current flows may vary substantially, which may cause inconsistent effects between individuals (Horvath et al. 2014). The variations of current flow may also be associated with paradoxical stimulation effects, as represented by "cathodal stimulation" and suppressing performance by either anodal or cathodal stimulation (Filmer et al. 2013). Moreover, a couple of recent reports suggest around half of healthy subjects do not show an expected excitatory effect on anodal tDCS (Lopez-Alonso et al. 2014; Wiethoff et al. 2014). Further, no persistent excitability changes during anodal, cathodal or sham stimulation have been reported, while polarity-independent after-effect was present (Santarnecchi et al. 2014).

Recently, high-definition tDCS (HD-tDCS), using an array of small electrodes (as small as those for electroencephalogram), has been investigated in the context of increasing the focality (Edwards et al. 2013) to reduce interpersonal variations (Miranda et al. 2013; Datta et al. 2012). To the best of our knowledge, clinical benefits of HDtDCS in psychiatric conditions has not yet been reported. Also, novel approaches with regard to types of electric currents have been studied, suggesting unique effects on neurons. Transcranial alternating current stimulation (tACS) uses fluctuating and alternating polarity of current with a certain frequency, instead of direct current, and is suggested to produce brain oscillations (Helfrich et al. 2014). Transcranial random noise stimulation (tRNS) is a special form of tACS, with a frequency spectrum characteristic of white noise (Terney et al. 2008). Head-to-head comparison of tDCS, tACS and tRNS for tinnitus suggests tRNS elicits the largest suppressive effect on its loudness (Vanneste et al. 2013).

In this review, we will focus on the current status of applications of the $1 x 1$ tDCS method to psychiatric conditions. 


\section{Review \\ Methods}

Although this is not a systematic review, we adopted following criteria for reproducibility. We searched MEDLINE using the words "transcranial direct current stimulation" OR "tDCS" to find 1859 articles on 24th, March, 2015. Among them, we set our inclusion criteria for clinical trials which 1) are written in English, 2) target depression, cognitive enhancement, or schizophrenia, 3) are sham-controlled, and 4) consist of more than one session. Our exclusion criteria were 1) animal studies and/ or 2) focusing only on online effects (change during tDCS). We also checked the references of these articles, as well as recent reviews and meta-analyses on these topics.

\section{Depression}

Among psychiatric conditions, depression has been most extensively studied, and seems most promising target, although evidence is not entirely convincing. In a large double-blind randomized controlled trial (RCT) implemented in Brazil, 120 depressed patients were randomized with a $2 \times 2$ factorial design to sertraline, a serotonin reuptake inhibitor used as antidepressant, or placebo, and active or sham tDCS (Brunoni et al. 2013). Compared to sham-tDCS groups, tDCS groups showed significantly greater improvements of depressive symptoms after 10 stimulations in 2 weeks. The benefit of tDCS was further indicated at the end of a 4 week extension period in which two stimulations (every other week) were administered (Brunoni et al. 2013).

A hypothesized rationale of treating depression with tDCS is that altered balance in cortical activities between left and right dorsolateral prefrontal cortex (DLPFC) is related to depressive symptoms (Grimm et al. 2008). Therefore, the left DLPFC or F3 area in the international 10-20 system for electroencephalography is the main target of anodal stimulation. On the other hand, where the cathodal electrode is placed depends on trials. For example, right supraorbital area is commonly used (Palm et al. 2012; Loo et al. 2010; Boggio et al. 2008; Fregni et al. 2006), while some studies adopt F4 (Brunoni et al. 2013; Blumberger et al. 2012) or F8 area (Loo et al. 2012) in the right hemisphere. Further, the number of stimulation sessions differs from 5 to 15, and the size of electrodes also varies. A recent meta-analysis reports these methodological heterogeneities among RCTs, while showing significant effect size (Hedges' g of 0.37, $95 \%$ confidence interval of 0.04-0.7) and significant odds ratios for response $(1.63,1.26-2.12)$ and remission $(2.50,1.26-2.49)$ as a whole (Shiozawa et al. 2014). Only 259 individuals in seven RCTs were evaluated in the meta-analysis; therefore it may be underpowered for statistical comparison in terms of methodological optimality, such as number of sessions, duration of one session, electrode placement, patient characteristics predicting good response, and so forth. In terms of electrode placement, a computational modeling study of tDCS for depression (Bai et al. 2014) suggests montages with the anodal electrode on frontal areas and the cathodal on occipital or extracellular areas may result in greater stimulation of deep structures, including anterior cingulate cortex. These authors suggest tDCS may be effective for melancholic depression, which deserves evaluations with RCTs.

Regarding safety, 8 out of aforementioned 10 RCTs reported adverse events (AEs) during tDCS. Most of them were mild-to-moderate, local and transient, e.g. headache, 
skin redness, tingling or itchiness. AEs related to psychiatric outcomes include suicide commitment in the sham group (Loo et al. 2010), transient hypomania, and euphoria in the active group (Loo et al. 2012) (one case for each event). tDCS has been reported considerably safe, and all studies met suggested safety criteria (Bikson et al. 2009). Although a large retrospective trial (Poreisz et al. 2007) and other trials suggest AEs related to tDCS seem minor and transient, we should be aware of unreported AEs, as not all RCTs admit systematic AE surveillance, for example by using questionnaires (Brunoni et al. 2011).

Given its safety and effectiveness for depressive symptoms, tDCS may have advantages as a treatment option in specific clinical settings. For example, its non-systemic and non-pharmacological approach may be suitable for patients susceptible to antidepressants, such as pregnant women (Vigod et al. 2014), frail elderly people, and individuals comorbid for neurological diseases. Even if its effect size may not be as large as those of the first-line therapies, the additive effect of tDCS may facilitate its usage as an augmentation treatment for depression before considering other brain stimulation methods, in view of its cost, portability and feasibility. For instance, three RCTs with concurrent cognitive control training have been reported, which were not included in the abovementioned meta-analysis. One of them found significant an antidepressive effect of tDCS (Segrave et al. 2014; Brunoni et al. 2014), while others (Vanderhasselt et al. 2015; Brunoni et al. 2014) did not (Table 2).

\section{Cognitive enhancement}

Studies on the efficacy for treating depressive symptoms simultaneously revealed tDCS may be benign or somehow beneficial for cognitive functions, e.g. several types of memory, executive function, attention, and fluency (Mondino et al. 2014; DemirtasTatlidede et al. 2013) (Table 3). This has promoted recent tDCS research targeting cognitive enhancement in other psychiatric conditions, although a recent systematic review is reluctant to conclude that tDCS improves cognition in depressive patients due to methodological issues and risk of biases (Tortella et al. 2014).

Two RCTs have been conducted for healthy subjects (Meinzer et al. 2014; Martin et al. 2013). A double-blind RCT recruiting 44 volunteers shows anodal stimulation

Table 2 RCTs of tDCS and CCT for depressed patients

\begin{tabular}{|c|c|c|c|c|}
\hline Author, Year & $\mathrm{N}$ & Arms & tDCS protocol & Results \\
\hline \multirow{3}{*}{$\begin{array}{l}\text { Segrave, } 2014 \\
\text { (Segrave et al. 2014) }\end{array}$} & \multirow{3}{*}{27} & $\mathrm{CCT}+\mathrm{tDCS}$ & 5 sessions in one week, & \multirow{3}{*}{$\begin{array}{l}\text { Significantly more change in } \\
\text { MADRS and BDI-II over time in } \\
C C T+\text { tDCS than others }\end{array}$} \\
\hline & & $\begin{array}{l}\text { Sham } C C T+ \\
\text { tDCS }\end{array}$ & $2 \mathrm{~mA}, 24 \mathrm{~min}, 35 \mathrm{~cm}^{2}$ & \\
\hline & & $\begin{array}{l}C C T+\text { sham } \\
\text { tDCS }\end{array}$ & A/C: F3/F8 & \\
\hline $\begin{array}{l}\text { Brunoni, } 2014 \\
\text { (Brunoni et al. 2014) }\end{array}$ & 37 & $\begin{array}{l}C C T+\mathrm{tDCS} \\
C C T+\text { sham } \\
\mathrm{tDCS}\end{array}$ & $\begin{array}{l}10 \text { sessions in two weeks, } \\
2 \mathrm{~mA}, 30 \mathrm{~min}, 25 \mathrm{~cm}^{2} \\
\text { Anode: } \mathrm{F} 3 \text { Cathode: } \mathrm{F} 4\end{array}$ & $\begin{array}{l}\text { Both groups show recovery after } \\
\text { four weeks and non-significantly } \\
\text { larger improvement of HDRS and } \\
\text { BDI in CCT + sham tDCS }\end{array}$ \\
\hline $\begin{array}{l}\text { Vanderhasselt, } 2015 \\
\text { (Vanderhasselt et al. 2015) }\end{array}$ & 33 & $\begin{array}{l}C C T+\text { tDCS } \\
C C T+\text { sham } \\
\text { tDCS }\end{array}$ & $\begin{array}{l}10 \text { sessions in two weeks, } 2 \\
\text { mA, } 30 \text { min, } 25 \mathrm{~cm}^{2} \text { Anode: } \\
\text { F3 Cathode: } \mathrm{F} 4\end{array}$ & $\begin{array}{l}\text { No significant difference in terms } \\
\text { of HDRS and BDI }\end{array}$ \\
\hline
\end{tabular}

CCT: cognitive control training, tDCS: transcranial direct current stimulation, $\mathrm{A} / \mathrm{C}$ : placement of anode/cathode electrodes, MADRS: Montgomery-Åsberg Depression Rating Scale, BDI: Beck depression inventory, HDRS: Hamilton Depression Rating Score 
Table 3 RCTs for healthy volunteers

\begin{tabular}{|c|c|c|c|c|}
\hline Author, Year & $\mathrm{N}$ & Arms & tDCS protocol & results \\
\hline $\begin{array}{l}\text { Meinzer, } 2014 \\
\text { (Meinzer et al. 2014) }\end{array}$ & 40 & $\begin{array}{l}\text { tDCS during learning } \\
\text { sessions sham tDCS } \\
\text { during learning sessions }\end{array}$ & $\begin{array}{l}5 \text { consecutive days, } 1 \\
\mathrm{~mA}, 20 \mathrm{~min}, 35 \mathrm{~cm}^{2} \\
\text { Anode: Cp5 Cathode: } \\
\text { right supraorbital region }\end{array}$ & $\begin{array}{l}\text { tDCS was significantly better } \\
\text { in learning and recognition in } \\
\text { new vocabularies and its benefit } \\
\text { was maintained at one-week } \\
\text { follow-up. }\end{array}$ \\
\hline $\begin{array}{l}\text { Martin, } 2013 \\
\text { (Martin et al. 2013) }\end{array}$ & 60 & $\begin{array}{l}\mathrm{tDCS} \text { sham } \mathrm{tDCS}+\mathrm{CT} \\
\mathrm{tDCS}+\mathrm{CT}\end{array}$ & $\begin{array}{l}10 \text { sessions in } 2 \text { weeks, } \\
2 \mathrm{~mA}, 30 \text { min, } 35 \mathrm{~cm}^{2} \\
\text { Anode: F3 Cathode: } \\
\text { right deltoid muscle }\end{array}$ & $\begin{array}{l}\text { No significant difference on } \\
\text { performance on the dual } \\
\text { n-back task between tDCS + } \\
C T \text { and sham } \mathrm{tDCS}+\mathrm{CT} \text { in post } \\
\text { completion and follow-up. }\end{array}$ \\
\hline
\end{tabular}

CT: cognitive training (adaptive dual n-back working memory training task)

over the left posterior temporo-parietal junction for 5 consecutive days enhanced verbal learning ability to a greater extent compared to sham-stimulation, which lasted during the 1 week follow-up period (Meinzer et al. 2014). The other with 60 participants suggests significantly better performance of an adaptive dual n-back task in active/sham tDCS + cognitive training (CT) groups than the tDCS-only group after 10 daily sessions. The active tDCS + CT group also showed significantly greater gains on a nontrained test of attention and working memory than the tDCS-only group. On the other hand, there were no significant differences throughout the study between active and sham tDCS groups (Meinzer et al. 2014). Although itching and tickling were observed, serious AEs have not been reported in either study.

As for Alzheimer's disease (AD), four double-blind RCTs (Ferrucci et al. 2008; Boggio et al. 2009; Boggio et al. 2012; Cotelli et al. 2014) were selected in a recent systematic review (Nardone et al. 2015). However, we excluded two of them (Boggio et al. 2009; Ferrucci et al. 2008), which investigated single-session effects. On the other hand, we noticed two other RCTs; one (Khedr et al. 2014) reports a significant improvement in the Mini-Mental Scale Examination score after 10 sessions of anodal or cathodal stimulations over left DLPFC in 34 AD patients . The other (Suemoto et al. 2014), employing 40 apathetic AD patients, investigated the effect of six sessions of tDCS over 2 weeks on apathy, and found the lack of a significant benefit on apathy and cognitive outcomes. As shown in Table 4, studies so far conducted are largely heterogeneous in methodology and outcomes, and the numbers of participants are small. Only one out of 4 RCTs we selected reported AEs systematically to find tingling and scalp burning were significantly more frequent in active tDCS (Suemoto et al. 2014).

One of the largest issues regarding tDCS for cognitive enhancement is that the majority of evidence currently available is based on data from single stimulation or online effects. A recent qualitative analysis suggests a single-session tDCS for healthy subjects did not show any cognitive effect (Horvath et al. 2015). A meta-regressions of 19 experiments from 8 studies, targeting the left DLPFC as anode site, showed faster response in the N-back task, a measure of working memory, in subjects administered tDCS (Brunoni and Vanderhasselt 2014). Among these studies including online tDCS, 18 out of 19 used the crossover design with single stimulation (Brunoni and Vanderhasselt 2014).

Another issue concerning cognitive effects of tDCS is the relation between electrode placement and targeted cognitive domains. A single-blind RCT with various tDCS montages showed that positioning and size of electrodes on both anode and cathode sides affected performance on a semantic fluency task (Penolazzi et al. 2013). 
Table 4 RCTs for AD patients

\begin{tabular}{|c|c|c|c|c|}
\hline Author, Year & $\mathrm{N}$ & Arms & tDCS protocol & Results \\
\hline $\begin{array}{l}\text { Boggio, } 2012 \\
\text { (Boggio et al. 2012) }\end{array}$ & $15^{*}$ & tDCS Sham tDCS & $\begin{array}{l}5 \text { sessions in a week, } 2 \\
\mathrm{~mA}, 30 \mathrm{~min}, 35 \mathrm{~cm}^{2} \\
\text { Anode: } \mathrm{T} 3 \text { and T4 Cathode: } \\
\text { right deltoid muscle }\end{array}$ & $\begin{array}{l}\text { Significant improvement of } \\
\text { visual recognition memory } \\
\text { but no significant effect on } \\
\text { MMSE, ADAS-Cog, VAT }\end{array}$ \\
\hline $\begin{array}{l}\text { Cotelli, } 2014 \\
\text { (Cotelli et al. 2014) }\end{array}$ & 36 & $\begin{array}{l}\text { tDCS + ICMT sham } \\
\text { tDCS + ICMT tDCS + } \\
\text { motor training }\end{array}$ & $\begin{array}{l}10 \text { sessions in } 2 \text { weeks, } 2 \\
\text { mA, } 25 \text { min, } 25 \mathrm{~cm}^{2} \text { Anode: } \\
\text { F3 Cathode: right deltoid } \\
\text { muscle }\end{array}$ & $\begin{array}{l}\text { No significant improvement } \\
\text { in memory except face-name } \\
\text { association task }\end{array}$ \\
\hline $\begin{array}{l}\text { Khedr, } 2014 \\
\text { (Khedr et al. 2014) }\end{array}$ & 34 & $\begin{array}{l}\text { tDCS (Anodal F3 } \\
\text { stimulation) tDCS } \\
\text { (Cathodal F3 } \\
\text { stimulation) sham } \\
\text { tDCS }\end{array}$ & $\begin{array}{l}10 \text { sessions in } 2 \text { weeks, } 2 \\
\mathrm{~mA}, 25 \mathrm{~min}, 24 / 100 \mathrm{~cm}^{2} \\
\text { Anode: } \mathrm{F} 3 \text { Cathode: right } \\
\text { supraorbital region }\end{array}$ & $\begin{array}{l}\text { Both anodal and cathodal } \\
\text { stimulation showed more } \\
\text { significant improvement in } \\
\text { MMSE than sham. Reduction } \\
\text { of P300 is also observed in } \\
\text { tDCS. }\end{array}$ \\
\hline $\begin{array}{l}\text { Suemoto, } 2014 \\
\text { (Suemoto et al. 2014) }\end{array}$ & 40 & tDCS sham tDCS & $\begin{array}{l}6 \text { sessions in } 2 \text { weeks, } 2 \\
\text { mA, } 30 \text { min, } 35 \mathrm{~cm}^{2} \text { Anode: } \\
\text { F3Cathode: right orbit }\end{array}$ & $\begin{array}{l}\text { No significant difference in } \\
\text { Apathy, as well as ADAS-Cog } \\
\text { and other cognitive tests }\end{array}$ \\
\hline
\end{tabular}

ICMT: individual computerized memory training, MMSE: Mini-Mental State Examination, ADAS-Cog: Alzheimer Disease Assessment Scale Cognitive Subscale, VAT: visual attention task

*: crossover

Additionally, a recent double-blind RCT with 40 healthy subjects reports 10 sessions of anodal stimulation on bilateral DLPFC combined with computer-assisted cognitive training significantly enhanced verbal working memory and digit span (Park et al. 2014). In that study, the enhancement of verbal working memory lasted for 4 weeks, suggesting the after-effects of tDCS (Park et al. 2014). Montages with multiple electrodes (HD-tDCS), aiming at more focal and multi-focal stimulation, may provide a more sophisticated strategy to determine the optimal disposition of electrodes. Available data are still insufficient, and further efforts, e.g. robust biological modeling and computerized simulation (Truong et al. 2014), may help solve these issues. Especially, we should be aware that precise modeling of the induced electrical fields at the level of the individual brain is desirable in order to better estimate biological effects.

\section{Schizophrenia}

In schizophrenia, limited information is available about the effect of tDCS, and so far, two sham-controlled RCTs have been reported (Mattai et al. 2011; Brunelin et al. 2012; Andrade 2013). The first study (Mattai et al. 2011) was implemented to assess the tolerability of tDCS when applied to 12 pediatric schizophrenia patients; 10 sessions of tDCS (20-min duration) with $2 \mathrm{~mA}$ current was well tolerated.

Results from another study (Brunelin et al. 2012) suggest tDCS may alleviate positive symptoms, especially hallucinations, as well as negative symptoms. In this study, 30 schizophrenia patients with medication-refractory auditory hallucinations were randomized to receive 20 min of tDCS or sham stimulation twice a day on 5 consecutive days. Left DLPFC was stimulated with the anodal electrode and cathode was placed over the left temporoparietal cortex to inhibit neural activities around Broca's area. The active group showed a mean improvement of $31 \%$, which is significant, as measured by the 7-point Auditory Hallucination Rating Scale (Hoffman et al. 2003). This effect lasted for 3 months, although hallucinations remained in all patients. Additionally, negative symptoms, as evaluated by the Positive and Negative Symptom Scale (PANSS) (Kay et al. 1987), were significantly 
improved with tDCS. Severe AEs were not reported, but all patients experienced a transient mild tingling or a slight itching sensation associated with the onset of stimulation.

While not sham-controlled, one RCT compared unilateral (anode on F3 and cathode on TP3) and bilateral (anode on F3/F4 and cathode on TP3/TP4) tDCS (Fitzgerald et al. 2014). In that study, 24 patients with schizophrenia or schizoaffective disorder were randomized, and 11 received bilateral and 13 received unilateral tDCS once a day for 15 sessions in 3 weeks. Neither group showed any significant improvement in psychotic symptoms, as measured by PANSS or the Scale for the Negative Symptoms (Andreasen 1982). The cognitive effect of unilateral or bilateral tDCS was not reported, although some cognitive assessments were conducted (Fitzgerald et al. 2014). No major adverse events were reported, but some patients experienced itchiness, headaches and non-specific site discomfort.

\section{Conclusions and recommendations}

We provided an overview on the results of relative long-term effects of tDCS on clinical symptoms. Needless to say, large RCTs with methodological sophistication, targeting several psychiatric conditions, are awaited. RCTs must follow the safety criteria (Bikson et al. 2009) and the common protocols are electrode sizes of $25-35 \mathrm{~cm}^{2}$ (generating densities ranging from $0.28-0.80 \mathrm{~A} / \mathrm{m}^{2}$ ) for up to $20-40 \mathrm{~min}$ (Brunoni et al. 2012).

For depression, DLPFC is a rational target and results seem promising. A computed modeling study suggests fronto-extracephalic or fronto-occipital montages may be more efficient to stimulate DLPFC than montages used in the majority of the current studies (Bai et al. 2014), which needs further verifications. Besides, daily stimulation is probably preferable because one investigation suggested it can induce significantly greater cortical excitability than tDCS every other day (Alonzo et al. 2012). The aforementioned meta-analysis suggests a larger number of sessions may be more effective (Shiozawa et al. 2014). Trials of tDCS as augmenting therapy should also be implemented for other diseases we have mentioned, and future trials may want to incorporate into technologies to reduce interpersonal variability including individual anatomical modeling, while we should also acknowledge its current technical issues. Further, considering negative results in some trials, identification of treatment-responsive characteristics of patients is critical.

Areas of cognitive enhancement and treatment of schizophrenia, which are less established in terms of effective methodology, merits further explorations, based on pathophysiology-driven hypotheses. Some studies are likely to adopt the methods used in depression trials without rationales, and findings were often not replicated. Since the duration of each session and the total amount of current flow cannot be changed much for safety reasons, we should primarily focus on establishing appropriate parameters. In fact, electrode placement (including HD-tDCS) and type (mode) of current flow (including tACS and tRNS) could be good candidates, as supported by electrophysiological modeling (Truong et al. 2014; Eichelbaum et al. 2014).

Abbreviations

AD: Alzheimer's Disease; AE: Adverse Event; CT: Cognitive Training; DLPFC: Dorso Lateral Prefrontal Cortex; ECT: Electroconvulsive Therapy; HD-tDCS: High-Definition Transcranial Direct Current Stimulation; PANSS: Positive and Negative Symptom Scale; RCT: Randomized Controlled Trial; SANS: the Scale for the Negative Symptoms; 
tACS: transcranial Alternating Current Stimulation; tDCS: transcranial Direct Current Stimulation; tRNS: transcranial Random Noise Stimulation.

\section{Competing interests}

The authors declare that they have no competing interests.

\section{Authors' contributions}

YY and TS wrote the draft. Both authors' read and approved the final manuscript.

\section{Acknowledgements}

This work is supported by grants Japan Society for the Promotion of Science Grant-in-Aid for Scientific Research (No. 26461761 and 26860958), Health and Labour Sciences Research Grants for Comprehensive Research on Disability, Health, and Welfare (H26-Seishin-Ippan-011), and Intramural Research Grant for Neurological and Psychiatric Disorders of NCNP (27-1).

The authors thank Dr. Mitsutoshi Okazaki for fruitful discussions.

\section{Author details}

'Department of Psychiatry, National Center Hospital, National Center of Neurology and Psychiatry, 4-1-1

Ogawa-Higashi, Kodaira, Tokyo 187-8553, Japan. ${ }^{2}$ Department of Clinical Research Promotion, National Center Hospital, National Center of Neurology and Psychiatry, Tokyo, Japan.

Received: 16 January 2015 Accepted: 1 June 2015

\section{Published online: 19 August 2015}

\section{References}

Alonzo A, Brassil J, Taylor JL, Martin D, Loo CK (2012) Daily transcranial direct current stimulation (tDCS) leads to greater increases in cortical excitability than second daily transcranial direct current stimulation. Brain stimulation 5(3):208-213. doi:10.1016/.j.brs.2011.04.006

Andrade C (2013) Transcranial direct current stimulation for refractory auditory hallucinations in schizophrenia. The Journal of clinical psychiatry 74(11):e1054-1058. doi:10.4088/JCP.13f08826

Andreasen NC (1982) Negative symptoms in schizophrenia. definition and reliability. Arch Gen Psychiatry 39(7):784-788

Bai S, Dokos S, Ho KA, Loo C (2014) A computational modelling study of transcranial direct current stimulation montages used in depression. Neurolmage 87:332-344. doi:10.1016/..neuroimage.2013.11.015

Bikson M, Datta A, Elwassif M (2009) Establishing safety limits for transcranial direct current stimulation. Clinical neurophysiology : official journal of the International Federation of Clinical Neurophysiology 120(6):1033-1034. doi:10.1016/j.clinph.2009.03.018

Blumberger DM, Tran LC, Fitzgerald PB, Hoy KE, Daskalakis ZJ (2012) A randomized double-blind sham-controlled study of transcranial direct current stimulation for treatment-resistant major depression. Frontiers in psychiatry 3:74. doi:10.3389/fpsyt.2012.00074

Boggio PS, Ferrucci R, Mameli F, Martins D, Martins O, Vergari M et al (2012) Prolonged visual memory enhancement after direct current stimulation in Alzheimer's disease. Brain stimulation 5(3):223-230. doi:10.1016/j.brs.2011.06.006

Boggio PS, Khoury LP, Martins DC, Martins OE, de Macedo EC, Fregni F (2009) Temporal cortex direct current stimulation enhances performance on a visual recognition memory task in Alzheimer disease. J Neurol Neurosurg Psychiatry 80(4):444-447. doi:10.1136/jnnp.2007.141853

Boggio PS, Rigonatti SP, Ribeiro RB, Myczkowski ML, Nitsche MA, Pascual-Leone A et al (2008) A randomized, double-blind clinical trial on the efficacy of cortical direct current stimulation for the treatment of major depression. The international journal of neuropsychopharmacology/official scientific journal of the Collegium Internationale Neuropsychopharmacologicum 11(2):249-254. doi:10.1017/S1461145707007833

Brunelin J, Mondino M, Gassab L, Haesebaert F, Gaha L, Suaud-Chagny MF et al (2012) Examining transcranial directcurrent stimulation (tDCS) as a treatment for hallucinations in schizophrenia. Am J Psychiatry 169(7):719-724. doi:10.1176/appi.ajp.2012.11071091

Brunoni AR, Amadera J, Berbel B, Volz MS, Rizzerio BG, Fregni F (2011) A systematic review on reporting and assessment of adverse effects associated with transcranial direct current stimulation. The international journal of neuropsychopharmacology/official scientific journal of the Collegium Internationale Neuropsychopharmacologicum 14(8):1133-1145. doi:10.1017/S1461145710001690

Brunoni AR, Boggio PS, De Raedt R, Bensenor IM, Lotufo PA, Namur V et al (2014) Cognitive control therapy and transcranial direct current stimulation for depression: a randomized, double-blinded, controlled trial. J Affect Disord 162:43-49. doi:10.1016/j.jad.2014.03.026

Brunoni AR, Nitsche MA, Bolognini N, Bikson M, Wagner T, Merabet L et al (2012) Clinical research with transcranial direct current stimulation (tDCS): challenges and future directions. Brain stimulation 5(3):175-195. doi:10.1016/j.brs.2011.03.002

Brunoni AR, Valiengo L, Baccaro A, Zanao TA, de Oliveira JF, Goulart A et al (2013) The sertraline vs. electrical current therapy for treating depression clinical study: results from a factorial, randomized, controlled trial. JAMA psychiatry 70(4):383-391. doi:10.1001/2013.jamapsychiatry.32

Brunoni AR, Vanderhasselt MA (2014) Working memory improvement with non-invasive brain stimulation of the dorsolateral prefrontal cortex: a systematic review and meta-analysis. Brain Cogn 86:1-9. doi:10.1016/j.bandc.2014.01.008

Cotelli M, Manenti R, Brambilla M, Petesi M, Rosini S, Ferrari C et al (2014) Anodal tDCS during face-name associations memory training in Alzheimer's patients. Front Aging Neurosci 6:38. doi:10.3389/fnagi.2014.00038

Datta A, Truong D, Minhas P, Parra LC, Bikson M (2012) Inter-individual variation during transcranial direct current stimulation and normalization of dose using MRl-derived computational models. Frontiers in psychiatry 3:91. doi:10.3389/fpsyt.2012.00091

DemirtasTatlidede A, VahabzadehHagh AM, PascualLeone A (2013) Can noninvasive brain stimulation enhance cognition in neuropsychiatric disorders? Neuropharmacology 64:566-578. doi:10.1016/J.Neuropharm.2012.06.020 
Edwards D, Cortes M, Datta A, Minhas P, Wassermann EM, Bikson M (2013) Physiological and modeling evidence for focal transcranial electrical brain stimulation in humans: a basis for high-definition tDCS. Neurolmage 74:266-275. doi:10.1016/..neuroimage.2013.01.042

Eichelbaum S, Dannhauer M, Hlawitschka M, Brooks D, Knosche TR, Scheuermann G (2014) Visualizing simulated electrical fields from electroencephalography and transcranial electric brain stimulation: a comparative evaluation. Neurolmage 101:513-530. doi:10.1016/j.neuroimage.2014.04.085

Ferrucci R, Mameli F, Guidi I, Mrakic-Sposta S, Vergari M, Marceglia S et al (2008) Transcranial direct current stimulation improves recognition memory in Alzheimer disease. Neurology 71(7):493-498. doi:10.1212/01.wnl.0000317060.43722.a3

Filmer HL, Mattingley JB, Dux PE (2013) Improved multitasking following prefrontal tDCS. Cortex; a journal devoted to the study of the nervous system and behavior 49(10):2845-2852. doi:10.1016/j.cortex.2013.08.015

Fitzgerald PB, McQueen S, Daskalakis ZJ, Hoy KE (2014) A negative pilot study of daily bimodal transcranial direct current stimulation in schizophrenia. Brain stimulation 7(6):813-816. doi:10.1016/j.brs.2014.08.002

Fregni F, Boggio PS, Nitsche MA, Rigonatti SP, Pascual-Leone A (2006) Cognitive effects of repeated sessions of transcranial direct current stimulation in patients with depression. Depression and anxiety 23(8):482-484. doi:10.1002/da.20201

George MS, Higgins ES, Nahas ZH (2011) Brain stimulation therapies: vagus nerve stimulation, transcranial magnetic stimulation, transcranial direct current stimulation, and deep brain stimulation. In: Coffey CE, Cummings JL (eds) The American psychiatric publishing textbook of geriatric neuropsychiatry. Third Edition, Amer Psychiatric Pub, pp 305-330

Grimm S, Beck J, Schuepbach D, Hell D, Boesiger P, Bermpohl F et al (2008) Imbalance between left and right dorsolateral prefrontal cortex in major depression is linked to negative emotional judgment: an fMRI study in severe major depressive disorder. Biol Psychiatry 63(4):369-376. doi:10.1016/j.biopsych.2007.05.033

Helfrich RF, Schneider TR, Rach S, Trautmann-Lengsfeld SA, Engel AK, Herrmann CS (2014) Entrainment of brain oscillations by transcranial alternating current stimulation. Current biology : CB 24(3):333-339. doi:10.1016/j.cub.2013.12.041

Hoffman RE, Hawkins KA, Gueorguieva R, Boutros NN, Rachid F, Carroll K et al (2003) Transcranial magnetic stimulation of left temporoparietal cortex and medication-resistant auditory hallucinations. Arch Gen Psychiatry 60(1):49-56

Horvath JC, Carter O, Forte JD (2014) Transcranial direct current stimulation: five important issues we aren't discussing (but probably should be). Front Syst Neurosci 8:2. doi:10.3389/fnsys.2014.00002

Horvath JC, Forte JD, Carter O (2015) Quantitative review finds no evidence of cognitive effects in healthy populations from single-session transcranial Direct Current Stimulation (tDCS). Brain stimulation. doi:10.1016/j.brs.2015.01.400

Kay SR, Fiszbein A, Opler LA (1987) The positive and negative syndrome scale (PANSS) for schizophrenia. Schizophr Bull 13(2):261-276

Khedr EM, Gamal NF, El-Fetoh NA, Khalifa H, Ahmed EM, Ali AM et al (2014) A double-blind randomized clinical trial on the efficacy of cortical direct current stimulation for the treatment of Alzheimer's disease. Front Aging Neurosci 6:275. doi:10.3389/fnagi.2014.00275

Kim JH, Kim DW, Chang WH, Kim YH, Kim K, Im CH (2014) Inconsistent outcomes of transcranial direct current stimulation may originate from anatomical differences among individuals: electric field simulation using individual MRI data. Neurosci Lett 564:6-10. doi:10.1016/j.neulet.2014.01.054

Loo CK, Alonzo A, Martin D, Mitchell PB, Galvez V, Sachdev P (2012) Transcranial direct current stimulation for depression: 3-week, randomised, sham-controlled trial. The British journal of psychiatry : the journal of mental science 200(1):52-59. doi:10.1192/bjp.bp.111.097634

Loo CK, Sachdev P, Martin D, Pigot M, Alonzo A, Malhi GS et al (2010) A double-blind, sham-controlled trial of transcranial direct current stimulation for the treatment of depression. The international journal of neuropsychopharmacology/ official scientific journal of the Collegium Internationale Neuropsychopharmacologicum 13(1):61-69. doi:10.1017/S1461145709990411

Lopez-Alonso V, Cheeran B, Rio-Rodriguez D, Fernandez-Del-Olmo M (2014) Inter-individual variability in response to non-invasive brain stimulation paradigms. Brain stimulation 7(3):372-380. doi:10.1016/j.brs.2014.02.004

Martin DM, Liu R, Alonzo A, Green M, Player MJ, Sachdev P et al (2013) Can transcranial direct current stimulation enhance outcomes from cognitive training? a randomized controlled trial in healthy participants. The international journal of neuropsychopharmacology/official scientific journal of the Collegium Internationale Neuropsychopharmacologicum 16(9):1927-1936. doi:10.1017/S1461145713000539

Mattai A, Miller R, Weisinger B, Greenstein D, Bakalar J, Tossell J et al (2011) Tolerability of transcranial direct current stimulation in childhood-onset schizophrenia. Brain stimulation 4(4):275-280. doi:10.1016/j.brs.2011.01.001

Medeiros LF, de Souza IC, Vidor LP, de Souza A, Deitos A, Volz MS et al (2012) Neurobiological effects of transcranial direct current stimulation: a review. Frontiers in psychiatry 3:110. doi:10.3389/fpsyt.2012.00110

Meinzer M, Jahnigen S, Copland DA, Darkow R, Grittner U, Avirame K et al (2014) Transcranial direct current stimulation over multiple days improves learning and maintenance of a novel vocabulary. Cortex; a journal devoted to the study of the nervous system and behavior 50:137-147. doi:10.1016/j.cortex.2013.07.013

Miranda PC, Mekonnen A, Salvador R, Ruffini G (2013) The electric field in the cortex during transcranial current stimulation. Neurolmage 70:48-58. doi:10.1016/j.neuroimage.2012.12.034

Mondino M, Bennabi D, Poulet E, Galvao F, Brunelin J, Haffen E (2014) Can transcranial direct current stimulation (tDCS) alleviate symptoms and improve cognition in psychiatric disorders? The world journal of biological psychiatry : the official journal of the World Federation of Societies of Biological Psychiatry 15(4):261-275. doi:10.3109/15622975.2013.876514

Nardone R, Holler Y, Tezzon F, Christova M, Schwenker K, Golaszewski S et al (2015) Neurostimulation in Alzheimer's disease: from basic research to clinical applications. Neurological sciences : official journal of the Italian Neurological Society and of the Italian Society of Clinical Neurophysiology. doi:10.1007/s10072-015-2120-6

Nitsche MA, Paulus W (2001) Sustained excitability elevations induced by transcranial DC motor cortex stimulation in humans. Neurology 57(10):1899-1901

Opitz A, Paulus W, Will S, Antunes A, Thielscher A (2015) Determinants of the electric field during transcranial direct current stimulation. Neurolmage 109:140-150. doi:10.1016/.neuroimage.2015.01.033 
Palm U, Schiller C, Fintescu Z, Obermeier M, Keeser D, Reisinger E et al (2012) Transcranial direct current stimulation in treatment resistant depression: a randomized double-blind, placebo-controlled study. Brain stimulation 5(3):242-251. doi:10.1016/j.brs.2011.08.005

Park SH, Seo JH, Kim YH, Ko MH (2014) Long-term effects of transcranial direct current stimulation combined with computer-assisted cognitive training in healthy older adults. Neuroreport 25(2):122-126. doi:10.1097/ WNR.00000000000000080

Penolazzi B, Pastore M, Mondini S (2013) Electrode montage dependent effects of transcranial direct current stimulation on semantic fluency. Behav Brain Res 248:129-135. doi:10.1016/.jbbr.2013.04.007

Poreisz C, Boros K, Antal A, Paulus W (2007) Safety aspects of transcranial direct current stimulation concerning healthy subjects and patients. Brain Res Bull 72(4-6):208-214. doi:10.1016/j.brainresbull.2007.01.004

Russell MJ, Goodman T, Pierson R, Shepherd S, Wang Q, Groshong B et al (2013) Individual differences in transcranial electrical stimulation current density. Journal of biomedical research 27(6):495-508. doi:10.7555/JBR.27.20130074

Santarnecchi E, Feurra M, Barneschi F, Acampa M, Bianco G, Cioncoloni D et al (2014) Time course of corticospinal excitability and autonomic function interplay during and following monopolar tDCS. Frontiers in psychiatry 5:86. doi:10.3389/fpsyt.2014.00086

Segrave RA, Arnold S, Hoy K, Fitzgerald PB (2014) Concurrent cognitive control training augments the antidepressant efficacy of tDCS: a pilot study. Brain stimulation 7(2):325-331. doi:10.1016/j.brs.2013.12.008

Shiozawa P, Fregni F, Bensenor IM, Lotufo PA, Berlim MT, Daskalakis JZ et al (2014) Transcranial direct current stimulation for major depression: an updated systematic review and meta-analysis. The international journal of neuropsychopharmacology/official scientific journal of the Collegium Internationale Neuropsychopharmacologicum 17(9):1443-1452. doi:10.1017/S1461145714000418

Stagg CJ, Lin RL, Mezue M, Segerdahl A, Kong Y, Xie J et al (2013) Widespread modulation of cerebral perfusion induced during and after transcranial direct current stimulation applied to the left dorsolateral prefrontal cortex. The Journal of neuroscience : the official journal of the Society for Neuroscience 33(28):11425-11431. doi:10.1523/JNEUROSCI.3887-12.2013

Stagg CJ, Nitsche MA (2011) Physiological basis of transcranial direct current stimulation. The Neuroscientist : a review journal bringing neurobiology, neurology and psychiatry 17(1):37-53. doi:10.1177/1073858410386614

Steinberg H (2013) Letter to the editor: transcranial direct current stimulation (tDCS) has a history reaching back to the 19th century. Psychol Med 43(3):669-671. doi:10.1017/S0033291712002929

Stroupe KT, Weaver FM, Cao L, Ippolito D, Barton BR, Burnett-Zeigler IE et al (2014) Cost of deep brain stimulation for the treatment of Parkinson's disease by surgical stimulation sites. Movement disorders : official journal of the Movement Disorder Society 29(13):1666-1674. doi:10.1002/mds.26029

Suemoto CK, Apolinario D, Nakamura-Palacios EM, Lopes L, Leite RE, Sales MC et al (2014) Effects of a non-focal plasticity protocol on apathy in moderate Alzheimer's disease: a randomized, double-blind, sham-controlled trial. Brain stimulation 7(2):308-313. doi:10.1016/j.brs.2013.10.003

Terney D, Chaieb L, Moliadze V, Antal A, Paulus W (2008) Increasing human brain excitability by transcranial high-frequency random noise stimulation. The Journal of neuroscience : the official journal of the Society for Neuroscience 28(52):14147-14155. doi:10.1523/JNEUROSCl.4248-08.2008

Tortella G, Selingardi PM, Moreno ML, Veronezi BP, Brunoni AR (2014) Does non-invasive brain stimulation improve cognition in major depressive disorder? A systematic review. CNS \& neurological disorders drug targets 13(10):1759-1769

Truong DQ, Huber M, Xie X, Datta A, Rahman A, Parra LC et al (2014) Clinician accessible tools for GUI computational models of transcranial electrical stimulation: BONSAI and SPHERES. Brain stimulation 7(4):521-524. doi:10.1016/j.brs.2014.03.009

Vanderhasselt MA, De Raedt R, Namur V, Lotufo PA, Bensenor IM, Boggio PS et al (2015) Transcranial electric stimulation and neurocognitive training in clinically depressed patients: a pilot study of the effects on rumination. Prog Neuro-Psychopharmacol Biol Psychiatry 57:93-99. doi:10.1016/j.pnpbp.2014.09.015

Vanneste S, Fregni F, De Ridder D (2013) Head-to-head comparison of transcranial random noise stimulation, transcranial AC stimulation, and transcranial DC stimulation for tinnitus. Frontiers in psychiatry 4:158. doi:10.3389/fpsyt.2013.00158

Vigod S, Dennis CL, Daskalakis Z, Murphy K, Ray J, Oberlander T et al (2014) Transcranial direct current stimulation (tDCS) for treatment of major depression during pregnancy: study protocol for a pilot randomized controlled trial. Trials 15(1):366. doi:10.1186/1745-6215-15-366

Wiethoff S, Hamada M, Rothwell JC (2014) Variability in response to transcranial direct current stimulation of the motor cortex. Brain stimulation 7(3):468-475. doi:10.1016/j.brs.2014.02.003

Woods AJ, Bryant V, Sacchetti D, Gervits F, Hamilton R (2014) Effects of electrode drift in transcranial direct current stimulation. Brain stimulation. doi:10.1016/j.brs.2014.12.007

\section{Submit your next manuscript to BioMed Central and take full advantage of:}

- Convenient online submission

- Thorough peer review

- No space constraints or color figure charges

- Immediate publication on acceptance

- Inclusion in PubMed, CAS, Scopus and Google Scholar

- Research which is freely available for redistribution

Submit your manuscript at www.biomedcentral.com/submit 